\title{
Building Information Systems Development Methods: Synthesising from a Basis in both Theory and Practice
}

\author{
Danielle C. Fowler \\ School of Information Technology, COTAR \\ Swinburne University \\ John St, Hawthorn, Victoria, 3122, Australia \\ dfowler@swin.edu.au
}

\author{
Paul A. Swatman \\ School of Management Information Systems \\ Deakin University \\ Burwood, Victoria, Australia \\ paul.swatman@deakin.edu.au
}

\begin{abstract}
In this paper, we discuss some methodological issues associated with research into requirements engineering, and describe the benefits afforded to us by using action research to explore issues associated with requirements elicitation, modelling and validation. FOOM is a requirements engineering method which is designed to facilitate the development of high-quality, requirements-conformant information systems. In creating FOOM, an overriding concern has been to ensure both theoretic soundness and practical applicability within the target domain. We discuss in this paper the benefits of using action research as an enquiry mechanism for exploring issues associated with requirements elicitation, modelling and validation, and the way in which it formed a central part of the method's evaluation and evolution.
\end{abstract}

\section{Introduction}

Poor quality systems and software development permeate the information systems industry. System development projects are notorious for being unpredictable and expensive, and for failing to meet requirements when they are delivered. A significant contributory factor in this is the Aequirements@problem. Within the software development community there is general agreement with Boehm (1976) view that most software errors are introduced during the specification stage and that it is much cheaper to fix problems during the early stages of software development than at the end (see e.g., Davis, 1994; McMorran 1993; Williams, 1994; Wordsworth, 1992).

Unfortunately, arriving at a complete and consistent specification is perhaps the hardest part of the software development task (Brooks, 1987). Nonetheless, identifying and stating the requirements, both accurately and precisely, is critical to producing good quality software. It is widely held that specification, or more generally requirements engineering, will continue to be a critical issue throughout the 1990s (Fraser, 1994; Siddiqi, 1994).

FOOM is an approach to high-quality requirements engineering which incorporates elements of the soft systems (Checkland 1981, 1991, 1995; Checkland and Scholes 1990) approach with formal specification and the object oriented paradigm. We were motivated to undertake the associated research programme by the pervasive dissatisfaction within industry of the performance of the information systems profession. We wanted to focus on developing a method applicable within the commercial and industrial, rather than scientific and technical, domain. In this paper, we discuss the influence our desire to make FOOM commercially relevant had on the method's theoretical development.

\section{Balancing theory and practice}

The structure of the FOOM development path was strongly influenced by our desire to develop an approach to requirements engineering which, while theoretically sound, was relevant to the commercial domain within which the requirements engineering would take place.

Requirements engineering is applied to a wide and heterogeneous range of problems. Of course, no single method may be optimal under all circumstances. As requirements engineering, whether viewed from an information systems or software engineering perspective, is an applied discipline, research in the area tends to fall into one of three common approaches:

Theory

Theoretical research, such as that done by Duke et al (1991) into building the Object Z specification language, provides sound theories and models for testing and use within industry. Theoretical research involves taking a (usually) small problem and investigating it with a thorough precision. Theoretical research is particularly important to a youthful discipline such as requirements engineering as models and frameworks without established theoretical ground cannot be relied 
upon. The problem is that much of what remains unknown in IS as a discipline relates to its complex social context, and theoretical research tends to focus on small, manageable problems which can be addressed in an empirical manner.

\section{Practice}

Whilst theoretical research is sufficient to investigate the natural sciences, much of what is poorly understood in IS as a discipline relates to its complex social context. Practice based research aims to identify or analyse critical issues from the natural domain of the area under study. The result is often a model of best practice derived from what is occurring in the field. The benefit of this approach is that models or frameworks are developed which are based soundly in the environment in which they are intended to be used, and are therefore (hopefully) more relevant. The disadvantage is that the models produced are not necessarily cohesive or comprehensive, and often are never tested before they are implemented: either because it is not of interest to the community concerned to investigate further what is considered acceptable, or because it is not possible to do so.

\section{Research and Development}

Research and development (R\&D) is concerned with the construction of artifacts in the course of pursuing a research question. There is, however, little literature in which this approach has been used with the explicit purpose of studying the application of the ISREM within the process.

Each of these approaches is valuable for investigating questions of a particular type but none is sufficient in itself. This is especially so in an area such as information systems requirements engineering where so many perceptions, embodied in its numerous reference disciplines, must be considered. Theories generated in an artificial environment run the risk of being irrelevant or not amenable to implementation; models derived from an applied context are not necessarily sound.

A common weakness of existing requirements engineering approaches is that their developers have concentrated solely on theoretic foundations, or on pragmatics, throughout their entire development process. The consequence of an unbalanced approach is that many requirements engineering methods are weak in one area or the other ${ }^{1}$ : either they lack the theoretic foundations to

Other methods (for example Soft Systems Methodology (Checkland, 1981) or VDM (Bjorner \& Jones, 1978) which have explicitly considered both theory and practice tend to have focused exclusively either on socio-organisational theory or on support continued refinement and their flaws become evident during systems design and implementation, or they are ill-suited for application within the commercial domain and their flaws become apparent during requirements elicitation or specification validation. We were interested in the middle ground: the marrying of theoretical strength with practical applicability. What we require therefore is a methodological approach which facilitates theoretical development within the intended setting.

For the FOOM project, we decided to use action research, an approach which has only latterly been adopted within the IS tradition, and which is not at all commonly used in Software Engineering. Action research has strong roots in sociology, psychology and education, disciplines which have long been grappling with the problem of conducting research into complex issues which often are not empirically measurable.

\section{Action research as an investigative mechanism}

The nature of action research is quite different to most research methods accepted within the Information Systems (and particularly the primarily positivist Computer Science and Software Engineering) discipline/s. Rather than hold the researcher to be a necessarily independent, unbiased observer of any given phenomenon, the action research approach acknowledges the difficulty in establishing researcher objectivity, and instead involves the researcher as a participant in the action and process of change (WoodHarper, 1985). The process involves the active and reflective involvement of the researcher in some purposeful action (Galliers, 1985).

Hult and Lennung (1980) offer this definition: Action research simultaneously assists in practical problem solving and expands scientific knowledge, as well as enhances the competencies of the respective actors, being performed collaboratively in an immediate situation using data feedback in a cyclical process aiming at an increased understanding of a given social situation, primarily applicable for the understanding of change processes in social systems and undertaken within a mutually acceptable framework. (Hult \& Lennung 1980, p 247).

mathematical/engineering-based theory. In other work, for example (Swatman \& Swatman, 1992a), we have argued that a broad and inclusive theoretic base is essential. 
In action research, the knowledge gained often results in a redefinition of the situation and of the problem. The result is a characteristically cyclic process of problem definition, action planning, implementation, data feedback and evaluation (Hult and Lennung, 1980) ${ }^{2}$ which is useful in the generation of theory (Jonsson, 1991): a primary consideration for us in choosing it as a research method. As well as collecting information, we expect some positive intervention in the research setting, which also provides the researcher with detailed knowledge of the situation being studied (Mumford et al, 1985). Amongst the benefits of using action research as an enquiry mechanism for ISREM evaluation and evolution, perhaps the most important are:

- $\quad$ support for the researcher to address issues associated with modelling which are notoriously intractable with other approachesCthe researchers, freed from the constraint of holding themselves separate from the research are therefore able to analyse and evaluate their involvement as a participant in the research cycle. This simplifies the difficulties of participant-researcher communication and is especially important in respect of requirements engineering issues such as elicitation of information and validation of models where such articulation may be ineffective

- the cyclical nature of the research approach, combined with this first person understanding of the problem, offers a richer body of data and potentially offers quicker and more effective theory building

- the ability to generate subjects for study: when evaluating a novel method, there are, generally, no examples of the use of the method in a commercial setting which, and no experts (other than the researchers) in the use of the method who, may be studied

Against these benefits, however, it is necessary to set the weaknesses of action research which include difficulties in generalising from outcomes which are both particular to the situation under study and clearly subjective.

The nature of action research most closely matched our requirements for, and the constraints on, the study of FOOM at the stage its development had reached in 1992: it was important to investigate a problem (that of producing high quality information systems) already existing in a real world setting, rather than taking a problem raised by theory and applying it in an artificial setting.

2 Susman and Evered (1978) divide the cyclic process into similar components---in their case: diagnosing, action planning, action taking, evaluating and specifying learning.

\section{The FOOM research programme}

The characteristics of FOOM have been discussed at greater length elsewhere (see Fowler et al. 1995; Swatman 1996), but briefly, FOOM stands upon three theoretic pillars:

Socio-organisational contextual analysis following the work of Checkland (1981, 1989, 1995) and Checkland \& Scholes (1990) which, in the general case, denies the existence of a single, objective requirements specification waiting to be discovered by the systems analyst

The object oriented approach in which situations are modelled as systems of interacting, encapsulated objects, each object belonging to some class

Mathematically formal specification languages in particular, the object oriented specification language Object-Z (Duke et al., 1991) by means of which the abstract characteristics of classes may be described precisely and unambiguously.

Very little data exists on the comparative effectiveness of the different systems development methods available. Evaluating an information systems development methodology (ISDM) is not only difficult, there is a paucity of literature on the topic of designing research programmes for the evaluation of ISDMs. Fitzgerald (1991) offers an insightful analysis of the information systems and software engineering literature, but finally is only able to offer suggestions

- that multiple research methods be used to strengthen an essentially subjective and qualitative evaluation; and

- that methodologists recognise explicitly the strengths and weaknesses of their research approaches.

In choosing the research method(s) to be used for FOOM, we accepted these suggestions and further recognised the benefits of the use of multiple methods in a cycle of theory building, testing and refinement $\mathrm{C}$ see, for example, Galliers (1988) and Jarvenpaa (1988). The appropriate research method depends inter alia on the state of the knowledge of the phenomena of interest. Since each research method has a different set of strengths and weaknesses, we use the method most appropriate for the research question or hypothesis under examination and for the state of our knowledge of the phenomena in general.

A number of research methods have been used within the overall FOOM research programme as it has evolved. The sequence of research phases and, for each phase, examples of the methods employed can be seen in figure 1 . 


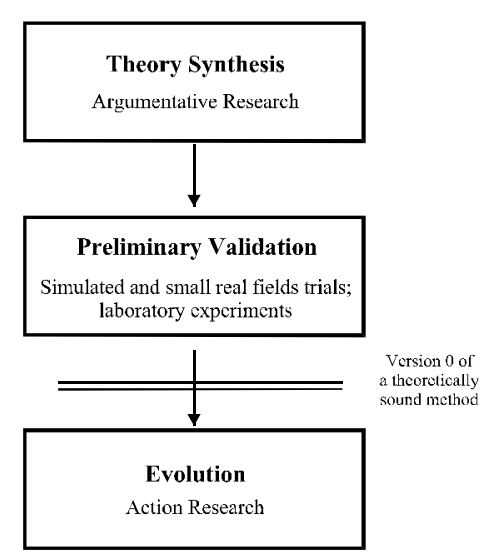

\section{Figure 1: Methodological Development in FOOM}

FOOM was originally developed by synthesis and logical argument, drawing on research in a number of largely independent areas across the breadth of the information systems and software engineering domains (Swatman \& Swatman, 1992a, 1992b). Preliminary evaluation of feasibility and potential benefit were undertaken by means of simulated (Swatman et al., 1991) case studies and small commercial (Swatman et al., 1992) system specifications; and by means of educational case studies and pseudo-laboratory experiments (Swatman, 1993). Some preliminary evaluation of FOOM as a framework (Fowler et al., 1993; Swatman, 1993; Swatman et al., 1992; Swatman \& Swatman 1992a) had suggested that a socio-organisational approach could be used beneficially in concert with object oriented formal specification techniques within the information systems domain.

Having established a theoretically sound method and shown that, in principle, the method could be applied beneficially by Information Systems professionals, our focus shifted toward demonstrating and enhancing the system in practice, in a commercial or industrial context. Given our desire to apply the FOOM framework in a commercial organisational setting, we had reduced our list of approaches to those appropriate for an in-depth analysis within such a setting. The poor suitability of the positivist perspective when dealing with a complex social setting, together with the formative nature of the research, eliminated quantitative methodology:

\footnotetext{
"when [quantitative] methods are used to investigate research topics about which ttheoretical development is scant or uncertain, research often is inefficient or
}

misleading. Either the power of deductive methods is underutilised, or theory and/or method are prematurely pressed into service when their underlying assumption cannot be met" (Bonoma, 1985, p.201).

We decided to adopt action research in order to strengthen the validation offered by earlier work, while retaining the opportunity for the expected necessary enhancement of the development method. Given that the earlier studies had established and tested (to an extent) the theoretic foundations of the method, it was important to test its practicality. The most important criterion, therefore, was to be able test the method in its intended setting .

Of the possible qualitative approaches, action research was chosen for its two main characteristics: it is useful in generating theory; and it seeks to produce practical solutions to problems in real world settings. These qualities were important in addressing issues in need of examination within the requirements engineering field. We were interested in elicitation and validation of requirements, and specifically in the process associated with modelling requirements. In order to build a method which incorporated formal techniques into the requirements specification phase (often utilised only further down the lifecycle), we needed to investigate how such modelling techniques might be used.

The advantage of using action research was that it enabled deeper insight to be gained into the modelling process being investigated while remaining within the natural setting of the phenomenon under study. Given the preliminary stage of the research and the lack of tested theory in the area, we required an approach which would be useful in generating ideas to test. The emergence of these ideas from the richer milieu in which the activities under study occur, rather than a controlled environment, offered the possibility of greater relevance.

Action research also offered another critical benefit: it facilitated us conducting research in an environment which otherwise would have been difficult to operate within. The literature on formal methods, the theory of which forms one pillar of the FOOM framework, suggest that formal specification techniques offer the systems development process considerable benefits in terms of ensuring clarity of thought and expression, and the communicative benefits associated with creating an unambiguous, concise statement of systems requirements about which we may formally reason. Formal methods, however, were widely considered by even the IS research community to be applicable only to computer scientists and the development of safety critical systems (eg Avison \& Fitzgerald). Given this perception, 
we expected the resistance to the trial of such methods in the commercial IS domain to be high.

We were faced with a dilemma: we could continue development of FOOM in simulated (educational) environments and hope that cases of IS developers using formal methods in industry would eventually increase. This would mean developing FOOM outside of its intended environment, with every chance that the method and associated tool support built would not be appropriate for its audience.

Very few people in industry possessed the requisite formal modelling skills, however, and it seemed unlikely at best that this might change without successful examples to encourage developers. It seemed clear we would need to change the perception of formal methods in industry in order to cultivate an environment in which to further develop FOOM. We were faced with the classic Catch 22: how to develop support for a new approach when people were reluctant to try it without a successful track record.

Given that it was not possible at that time to observe developers in industry using formal specification techniques, we would need to act as specifiers developing specifications within any cooperating organisation. We were left also with the issue of acceptance. The benefit of using action research was that we could proactively demonstrate the advantages of the approach to any participating organisation, and tailor the specifications developed to fit their business requirements. Mutual goal achievement is a primary objective of action research, unlike other research methods which focus on keeping observer and participant isolated, with the participant ideally having no directed effect on the research.

For us, this collaboration was essential if we were to be able to refine and evaluate FOOM in a realistic setting. While we were successful in finding a very supportive and committed organisation as a host for the action research study we undertook, we recognised that the chances of convincing numerous organisations to participate in case studies was remote at best, and their commitment would likely have been less than in the case of the single supportive organisation.

\section{The effect of method on results}

The use of action research in FOOM's development is shown in more detail in figure 2. Checkland (1995) argues persuasively that for a piece of action research to be more than anecdotal evidence, an intellectual framework within which knowledge in the research situation will be defined must be constructed, so that it may be appraised. Figure 2, modelled after that used by Checkland (1991,1995), shows the components of the research cycle used to further develop FOOM and their cyclical interaction.

The figure shows $(\mathrm{M})$, the developing method FOOM, based on the original framework of ideas (F) proposed in Swatman (1992). FOOM is used to tackle real world problem situations in the application area (R), the commercial information systems (particularly IOS) domain. Reflection based on this application process resulted in learning about the original framework, the developing method, and the domain of application. The result of the action research programme was a modified FOOM process model, shown in figure 3.

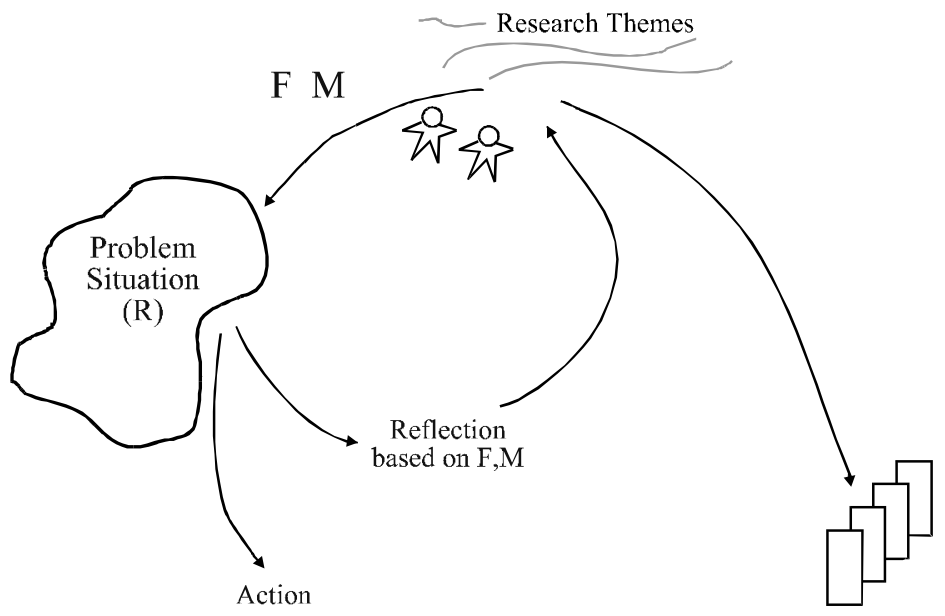

Figure 2: The (Action)Research Cycle

As can be seen in figure 3, FOOM involves a number of types of modelling in the requirements specification process. The appropriate modelling technique tends to progress from the primarily informal to the formal as the problem situation becomes more clearly understood. The end result of this process is the production of a formal statement of the requirements of the system which may then be used as a precise communication medium between the analysts and the system builders, or between the organisation and outsourced consultants, or in the case of purchasing an existing package.

We had assumed in developing the initial FOOM process model, that object oriented requirements modelling was a top down activity, in the sense that informal modelling would be completed, then used as the basis for developing a formal model of the system. This expectation was consistent with the requirements engineering literature. We discovered this was a very simplistic view of the modelling process. 


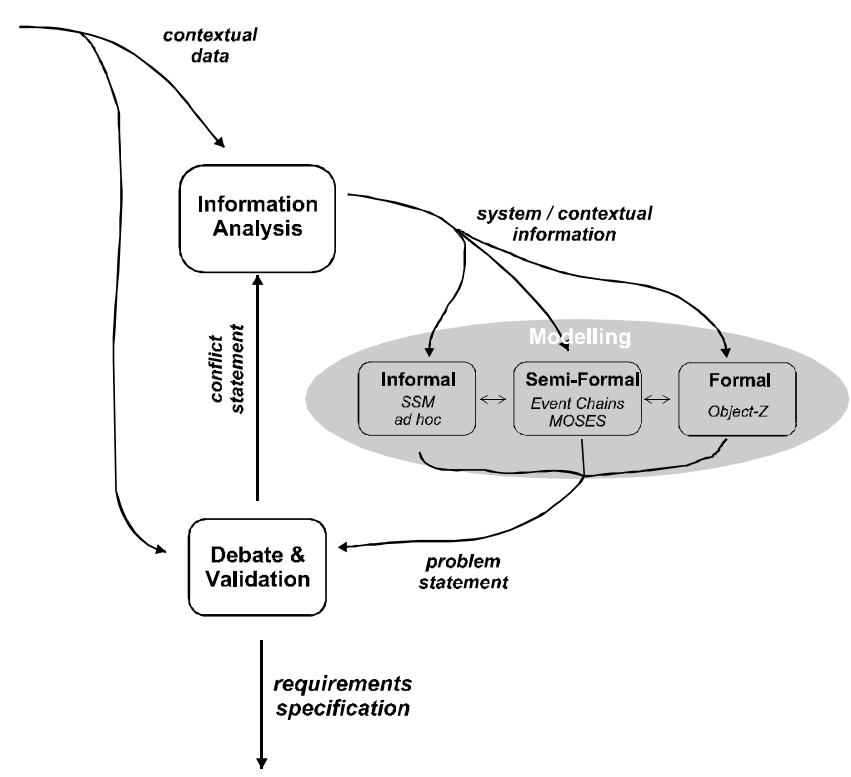

Figure 3. The modelling process cycle.

It is clearly difficult to analyse the modelling process. An internal thought process, modelling is not observable, and people, particularly it seems expert developers, are not good at articulating the thought processes involved. The benefit of being both modeller and researcher, observer and participant, is that this internal process may be analysed with much greater depth of understanding. The modifications made to the FOOM process model, which reflect our changed understanding of the relationship between formal and informal specification techniques and modelling processes, were a direct result of using action research as the investigative mechanism.

Consideration of these issues led to the examination and testing of the theoretic underpinnings of the method. The application of the method itself yielded insights into the practical suitability of the method. Work on FOOM has now progressed to a stage where other research methods are being incorporated into the overall FOOM research cycle. Ongoing work is involved with taking the broad qualitative analysis undertaken in the action research and selecting smaller, discrete areas of theory for which empirical testing (laboratory experiments) is suitable. Further qualitative research in the form of field trials are also continuing, to further validate the suitability of the method for requirements engineering in the commercial IS arena.

\section{Conclusion}

Building and evaluating information systems development methodologies (ISREMs) is a particularly difficult task, which has consequently not been done well within the information systems and software engineering communities.

Many of the studies looking at requirements engineering issues have focused on empirical approaches, which suffer from several disadvantages:

- in order to be manageable, they tend to be too small in scope to be representative

- it is hard to get experienced analysts to participate in such studies, and hard to get them to articulate their views

Students are frequently used as surrogates in requirements engineering studies, however there is no evidence to suggest that students tackling toy examples are representative of professional practice, or that such studies lead to the development of methods which scale up to a real world environment.

The objective of the FOOM research programme is to produce a high quality approach to requirements engineering which has a sound basis in both theory and practice. The adoption of action research for part of FOOM's development has aided us in this goal by allowing us to generate insight into requirements engineering based in the natural setting of the research. We believe some of the newer, interpretivist research methods such as action research offer the requirements engineering discipline great advantages in investigating issues not easily measured in an empirical manner, and should be considered more often.

\section{References}

[1] Avison D. E. \& Fitzgerald G. (1988) Information systems development: current themes and future directions. Information and Software Technology 30(8) 458-466.

[2] Bjorner D. \& Jones C.B. (eds) (1987) The Vienna Development Method: The Meta-Language, Springer Verlag, Berlin.

[3] Boehm B.W. (1976) Software engineering. IEEE Transactions on Computers C-25(12) 1226B1241.

[4] Bonoma T.V. (1985) Case research in marketing: Opportunities, problems and a process. Journal of Marketing Research 22 199B208.

[5] Brooks F. (1987) No silver bullet: Essence and accidents of software engineering. Computer 10B18.

[6] Checkland P.B. (1981) Systems Thinking, Systems Practice, Wiley, Chichester. 
[7] Checkland P.B. (1989) Soft systems methodology. Human Systems Management 8(4) 237B289.

[8] Checkland P.B. (1981) Systems Thinking, Systems Practice, Wiley, Chichester.

[9] Checkland P.B. (1989) Soft systems methodology. Human Systems Management 8(4) 237B289.

[10] Checkland P.B. (1991) From framework through experience to learning: the essential nature of action research. In Nissen H., Klein H. \& Hirschheim R. (eds) (1991) Information Systems Research: Contemporary Approaches and Emergent Traditions, NorthBHolland, Amsterdam.

[11] Checkland P.B. (1995) Soft systems methodology and its relevance to the development of information systems. In Stowell F. A. (1995) Information Systems Provision: the Contribution of Soft Systems Methodology, McGraw-Hill Book Company Europe, Berkshire, England.

[12] Checkland P.B. and Scholes J. (1990) Soft Systems Methodology in Practice, Wiley, Chichester.

[13] Davis A. (1993) Software Requirements: Objects, Functions and States, Prentice Hall, Englewood Cliffs.

[14] Duke R., King P., Rose G. and Smith G. (1991) The Object-Z specification language, Technical Report 91-1, Software Verification Research Centre, Department of Computer Science, University of Queensland, Australia.

[15] Fitzgerald G. (1991) Validating new information systems techniques: A retrospective analysis, in Nissen H.E. \& Hirschheim R. (eds), Information Systems Research: Cantemporary Approaches and Emergent Traditions, Elsevier Science Publishers B. V. (North Holland) 657B672.

[16] Fowler D.C., Swatman P.A. and Swatman P.M.C. (1993) Implementing EDI in the public sector: Including formality for enhanced control. In Gricar J. and Novak J. (editors) Strategic Systems in the Global Economy of the 90s: The 6th International Conference on Electronic Data Interchange and Interorganizational Systems, Moderna Organizacija, Kranj, Slovenia 244 B256.

[17] Fowler D.C. Swatman P.A. \& Wafula E. (1995) Formal methods in the IS domain: introducing a notation for presenting Object-Z specifications, Object Oriented Systems 2(2).

[18] Fraser M., Kumar K. and Vaishnavi V. (1994) Strategies for incorporating formal specifications in software development. Communications of the ACM, 37(10) 74 B86.

[19] Galliers R. D. (1985) In search of a paradigm for information systems research. In Mumford E. et al. (Eds) Research Methods in Information Systems, Elsevier Science Publishers B. V., Amsterdam, 281-297.

[20] Galliers R. D. and Land F. F. (1988) Authors response to Jarvenpaa (1988) below.
[21] Hult M. and Lennung S. (1980) Towards a definition of action research: a note and bibliography. The Journal of Management Studies, 241 B250.

[22] Jarvenpaa S. L. (1988) The importance of laboratory experimentation in IS research. Communications of the ACM 31(12) $1502 \mathrm{~B} 1504$.

[23] Jonsson S. (1991) Action Research, IFIP, Elsevier Science Publishers, 371-396.

[24] McMorran M. and Powell S. (1993) Z Guide for Beginners, Blackwell Scientific Publications, Oxford.

[25] Mumford E. et al. (Eds) Research Methods in Information Systems, Elsevier Science Publishers B. V., Amsterdam 281-297.

[26] Siddiqi J. (1994) Challenging universal truths of requirements engineering. IEEE Software, 11(2) 18 B19.

[27] Susman G. I. and Evered R. D. (1978) An assessment of the scientific merit of action research. Administrative Science Quarterly 23, 582-603.

[28] Swatman P.A. (1993) Using formal specifications in the acquisition of information systems: Educating information systems professionals. In Bowen J.P. and Nicholls J.E. (editors) Z User Workshop: London 1992. Workshops in Computing, Springer Verlag, London 205B239.

[29] Swatman P.A. (1996) Formal Object-Oriented MethodCFOOM. In Kilov H. and Harvey W. (Eds.), Specification of Behavioural Semantics in Object-Oriented Information Systems, Kluwer Academic Publishers, Norwell, Massachusetts.

[30] Swatman P.A. \& Swatman P.M.C. (1992a) Formal specification: An analytic tool for (management) information systems. Journal of Information Systems, 2(2) 121B160.

[31] Swatman P.A. and Swatman P.M.C. (1992b) Managing the formal specification of information systems. Proceeding of the International Conference on Organization and Information Systems, Bled, Slovenia.

[32] Swatman P.A., Swatman P.M.C. and Duke R. (1991) Electronic Data Interchange: A high -level formal specification in Object-Z. In Bailes P.A. (editor) 6th Australian Software Engineering Conference (ASWEC'91): Engineering Safe Software, Sydney, NSW.

[33] Swatman P.A., Fowler D.C. and Gan C.Y.M. (1992) Extending the useful application domain for formal methods. In Nicholls J.E. (editor) Z User Workshop: York 1991. Workshops in Computing, Springer Verlag, London.

[34] Williams L. (1994) Assessment of safety -critical specifications. IEEE Software, 11(1) 51B60. 
[35] Wordsworth J. B. (1993) Software Development with Z: A Practical Approach to Formal Methods in Software Engineering, Addison-Wesley.
[36] Wood-Harper A.T., Antill L. and Avison D. (1985) Information systems definition: the multiview approach, Blackwell Scientific Publications, Oxford. 